\title{
Zimbabwe: Les méthodes de dépistage des IAR chez les femmes sont chères et inefficaces
}

Population Council

Follow this and additional works at: https://knowledgecommons.popcouncil.org/departments_sbsr-rh

Part of the Demography, Population, and Ecology Commons, International Public Health Commons, and the Women's Health Commons How does access to this work benefit you? Let us know!

\section{Recommended Citation}

"Zimbabwe: Les méthodes de dépistage des IAR chez les femmes sont chères et inefficaces," FRONTIERES Résumés de Recherche Opérationnelle. Dakar: Population Council, 1999. 


\title{
Zimbabwe Les méthodes de dépistage des IAR chez les \\ Infections \\ femmes sont chères et inefficaces
}

l'Appareil Reproductif

$N^{\circ} 1$

\author{
Les méthodes courantes de dépistage des infections de l'appareil \\ reproductif chez les clientes des services de planification familiale sont \\ chères et inefficaces : les tests de laboratoire sont très coûteux, et la prise \\ en charge syndromique est souvent incapable de mettre certaines \\ infections en évidence et conduit à des traitements inutiles. Les \\ programmes de santé doivent continuer à privilégier les mesures \\ préventives : changement de comportement individuel et promotion de \\ l'utilisation des préservatifs.
}

\section{Contexte}

Les Infections de l'Appareil Reproductif (IAR) sont courantes au Zimbabwe. Beaucoup d'IAR augmentent le risque d'infection au Virus de l'Immunodéficience Humaine (VIH). En 1998, le Conseil National de Planification Familiale du Zimbabwe (ZNFPC) a entrepris une Recherche Opérationnelle afin d'évaluer la faisabilité de l'intégration du diagnostic des IAR et des services de traitement dans son menu de services. La population étudiée consistait en 1634 clientes de trois cliniques du ZNFPC. Chaque cliente a été interrogée sur les douleurs au bas ventre, les pertes vaginales, et autres symptômes d'IAR. Elles ont ensuite été examinées pour des signes cliniques d'IAR, et ont subi un test de laboratoire afin de confirmer la précision du diagnostic basé sur les symptômes et signes.

\section{Résultats}

- La prévalence des IAR. Les tests de laboratoire ont révélé que 9 pourcent de l'ensemble des clientes en Planification Familiale (PF) étaient atteintes d'une ou de plusieurs IAR sexuellement transmises (gonococcies, trichomonas, et chlamydiae). Ces trois IAR ont des conséquences graves pour la santé publique. La majorité des clientes souffrant d'IAR (26 pourcent du total) étaient affectées soit par une candidose soit par une vaginite bactérienne qui ne sont pas sexuellement transmissibles. Deux tiers des clientes en PF n'étaient atteintes d'aucune des cinq IAR décelées par les tests de laboratoire.

\section{- Application des directives cliniques.}

L'étude a évalué l'utilisation des directives nationales pour le diagnostic et le traitement des IAR lorsque des tests de laboratoires ne sont pas disponibles. Intitulées «prise en charge syndromique», ces directives donnent pour instruction aux prestataires, de traiter avec le même remède, toutes les causes communes d'un syndrome spécifique ou la combinaison de symptômes signalés par les clientes, ainsi que les signes cliniques observés durant un examen pelvien. L'identification des IAR sur la base de ces directives ne s'est pas révélée efficace parce que :

\& Les symptômes n'ont pas de corrélation avec les IAR. Plus d'un tiers des clientes atteintes d'une ou de plusieurs IAR (détectées par le laboratoire) n'avaient aucun symptôme, et par conséquent l'approche de la prise en charge syndromique n'a pas été en mesure de diagnostiquer les infections. En revanche, 47 pourcent des clientes en PF qui manifestaient des symptômes et des signes cliniques d'IAR, et qui par conséquent étaient identifiées comme étant infectées selon la méthode de la prise en charge syndromique, n'étaient en réalité atteintes d'aucune des cinq IAR objet du test. 
$\leftrightarrow$ Les prestataires de service ne suivaient pas toujours les directives de la prise en charge syndromique. Ils n'ont traité que 53 pourcent des femmes qui se plaignaient de douleur au bas ventre et 65 pourcent de celles qui ont fait part de pertes vaginales, et chez qui les signes cliniques avaient été décelés. Les autres n'ont bénéficié d'aucun traitement (certaines n'étaient pas infectées et n'avaient donc pas besoin de traitement).

\section{- Coût des interventions. Aucune des} interventions étudiées n'est abordable pour les programmes dans les zones à faible revenu. L'intervention la moins chère consiste à utiliser la prise en charge syndromique pour ne consulter que les clientes en PF qui sollicitent des services en IAR (voir tableau). Cependant, cette intervention a ses inconvénients. Dans cette étude, 75 pourcent des cas d'IAR n'ont pas été décelés, pendant que 56 pourcent des femmes traitées avaient été considérées à tort comme étant infectées et avaient reçu un traitement dont elle n'avaient pas besoin. Les tests de laboratoires aboutissent à des diagnostics fiables, mais ils sont très onéreux.

\section{Implications pour les programmes}

- La majorité des structures de santé des pays en développement ne disposent pas des ressources nécessaires à la mise en œuvre de la prise en charge syndromique à grande échelle. Au Zimbabwe par exemple, les dépenses de santé par habitant étaient estimées en 1998 à 47\$ US. L'application de la prise en charge syndromique des IAR à tous les clientes PF utiliserait plus de 10 pourcent de ces maigres ressources.

- L'inefficacité de l'approche de la prise en charge syndromique de l'identification des femmes atteintes d'IAR appelle à des efforts concertés en plaidoyer pour un appui au développement de tests de laboratoire plus simples et plus économiques.

- En l'absence d'approches plus économiques de la prise en charge syndromique, le ZNFPC et d'autres structures de santé doivent mettre encore plus l'accent sur les mesures destinées à réduire les rapports sexuels à risque ou non protégés, au nombre desquels il y a la promotion du préservatif et les services de counseling afin de renforcer chez les clientes la perception du risque personnel et une bonne connaissance des comportements les plus sûrs.

Décembre 1999

\begin{tabular}{|l|c|c|c|c|}
\hline \multicolumn{1}{|c|}{$\begin{array}{c}\text { Modèles de diagnostic } \\
(\mathrm{n}=1623)\end{array}$} & $\begin{array}{c}\text { \# de femmes } \\
\text { correctement } \\
\text { traitées }\end{array}$ & $\begin{array}{c}\text { \# de femmes } \\
\text { qui ont } \\
\text { inutilement } \\
\text { reçu des } \\
\text { remèdes }\end{array}$ & $\begin{array}{c}\text { Coût total } \\
\text { des remèdes } \\
\text { et tests de } \\
\text { laboratoire } \\
\text { (USD) }\end{array}$ & $\begin{array}{c}\text { Coût par } \\
\text { cliente de } \\
\text { clinique } \\
\text { (USD) }\end{array}$ \\
\hline $\begin{array}{l}\text { Approche syndromique pour } \\
\text { les clientes sollicitant des } \\
\text { services IAR (n=410) }\end{array}$ & 130 & 168 & 4.024 & 2.48 \\
\hline $\begin{array}{l}\text { Approche syndromique pour } \\
\text { toutes les clientes }\end{array}$ & 337 & 298 & 8.605 & 5.30 \\
\hline $\begin{array}{l}\text { Approche syndromique pour } \\
\text { toutes les clientes avec tests } \\
\text { de laboratoire pour les clientes } \\
\text { manifestant des symptômes } \\
\text { d'IAR et des signes cliniques }\end{array}$ & 337 & 0 & 6.722 & 10.30 \\
\hline $\begin{array}{l}\text { Tests de laboratoire pour } \\
\text { toutes les clientes PF }\end{array}$ & & & & \\
\hline
\end{tabular}

Zimbabwe National Family Planning Council. 1999. « Demande pour et rentabilité de l'intégration de services IAR/VIH et des services cliniques PF au Zimbabwe ». Pour de plus amples informations contacter: Population Council, P.O. Box 17643, Nairobi, Kenya. Tel. : 254-2-713-480 ; Fax : 254-2-713-479 ; E-mail: publications@popcouncil.or.ke.

Ce projet a été réalisé avec le concours de L'AGENCE AMERICAINE POUR LE DEVELOPPEMENT INTERNATIONAL

(USAID) aux termes du contrat numéro CCP-3030-C-00-3008-00 et de l'accord de coopération numéro HRN-A-00-98-00012-00. 